\section{DISCUTIENDO LA NEUTRALIDAD DE LA RED: DE LOS DISCURSOS DOMINANTES A LAS PRÁCTICAS EN CONTEXTOS PERIFÉRICOS}

\author{
Mariano Zukerfeld \\ Universidad de Buenos Aires, Argentina \\ marianozukerfeld@e-tcs.org
}

\section{DEBATING NET NEUTRALITY: FROM DOMINANT DISCOURSES TO PRACTICES IN PERIPHERAL CONTEXTS}

\author{
Bernadette Califano \\ Universidad de Buenos Aires, Argentina \\ bernacali@gmail.com
}

\begin{abstract}
Para citar este artículo:
Zukerfeld, M., \& Califano, B. (2019). Discutiendo la neutralidad de la red: de los discursos dominantes a las prácticas en contextos periféricos. Commons. Revista de Comunicación y Ciudadanía Digital, 8(1), 5-43.

http://dx.doi.org/10.25267/COMMON.2019.v8.i1.01
\end{abstract}

Fecha de recepción: 15/02/2019. Fecha de aceptación: 25/03/2019

\section{Resumen}

Este trabajo se propone discutir los discursos dominantes sobre la neutralidad de la red (NN). Su argumento central es que el funcionamiento de internet no guarda relación con los discursos dominantes que la asocian con un tratamiento igualitario de los paquetes de información que circulan a través de las redes, aún allí donde la NN se encuentra vigente. El artículo analiza cinco situaciones en las que se verifica un tratamiento dispar de los paquetes de información: las prácticas de gestión del tráfico en internet; la existencia de redes de distribución de contenidos; la priorización que realizan los buscadores; la discriminación geográfica en términos de velocidades de conexión entre países, y las diferencias entre las velocidades de carga y descarga.

\begin{abstract}
This paper is aimed at discussing dominant discourses regarding the so-called Net Neutrality (NN). In its most diffused definition $N N$ is associated with equal treatment to all packages of information, independently from any variable regarding internauts who send and receive those packages. Our main argument is that such NN is hardly verified in actual internet functioning, even in countries where NN laws have been passed and enforced. This argument is unfolded through analyzing five empirical situations where packages receive an unequal treatment: traffic management, Content Delivery Networks, prioritization performed by search engines, differences regarding Internet speed between countries, and between upload and download, configuring a discrimination against peripheral countries.
\end{abstract}

\section{Keywords}

Communication rights, ideological discourses, internet, net neutrality, public policies 


\section{Introducción}

La noción de neutralidad de la red ha sido ampliamente discutida en los ámbitos vinculados al desarrollo de internet y las tecnologías digitales en relación con el devenir político, económico y cultural de las sociedades actuales. Sin embargo, en los medios de comunicación e incluso en algunos campos académicos de las ciencias sociales (especialmente en contextos periféricos como los de las sociedades latinoamericanas) el consenso a favor de los principios que se asocian a la neutralidad de la red es tan abrumador que el debate se ha visto en buena medida clausurado, quizás antes de tiempo. Así, se ha aceptado que la neutralidad de la red tal y como se la ha caracterizado y definido en los contextos que han acuñado, estabilizado el término y difundido su operacionalización, es la mejor alternativa en términos éticos, políticos y económicos para todos los países y regiones del mundo.

Este trabajo ha sido concebido como parte de una investigación más amplia respecto a la regulación de internet en contextos periféricos entendida como política de desarrollo ${ }^{1}$, partiendo de la situación puntual de la Argentina. La neutralidad de la red, que reúne un conjunto de principios regulatorios (por acción u omisión), se perfila como una temática central en ese marco. Ahora bien, ¿funciona la neutralidad de la red en la práctica del modo en que los discursos dominantes lo indican? El discurso sobre la neutralidad de la red, ¿es en sí mismo neutral o disimula intereses en pugna? ¿Están contemplados los intereses de los países periféricos (distintos actores) como la Argentina en esos discursos y regulaciones? En el presente artículo nos proponemos responder a la primera de esas preguntas y comentar brevemente las restantes.

1. Con ello nos referimos a políticas públicas que apunten a incrementar las capacidades productivas e innovadoras nacionales, teniendo en cuenta el escenario geopolítico y el contexto global. En línea con las corrientes heterodoxas dentro de teorías de desarrollo económico, se entiende que las estructuras productivas con alto dinamismo tecnológico tienen altas posibilidades desarrollo a largo plazo (cfr. Schteingart \& Coatz, 2015). 
Para ello, el trabajo apela a una estrategia metodológica cualitativa que recurre a la técnica del análisis bibliográfico, a fin de revisar la literatura y los discursos existentes sobre el tema, a entrevistas a expertos y al análisis cuantitativo de datos secundarios, con el objetivo de responder a la pregunta de investigación.

El texto está organizado del siguiente modo. Esta introducción funciona como primera sección. La segunda se ocupa de dos aspectos. Por un lado, de describir los orígenes del debate en torno a la neutralidad de la red y de presentar dos tipos de definiciones que lo vertebran: una restrictiva de índole técnicoeconómica y otra más amplia, asociada a ideas de igualdad y libertad y de carácter político-cultural. Por el otro, de señalar dos tipos de discurso respecto a esta noción, que responden a intereses de dos grupos de actores en pugna: los del nivel de infraestructura de internet (ISPs, proveedores de conexión, carriers, etc.) de un lado, y los de los niveles de contenidos y software (usualmente llamados proveedores de contenidos y servicios), de otro. Ello nos permitirá demostrar que estos últimos actores realizan una notable operación retórica consistente en no considerar transgresiones a la neutralidad de la red a aquellas infracciones que son contrarias a sus intereses comerciales. En la tercera sección intentamos sistematizar situaciones en las que la neutralidad de la red (en su acepción dominante) no se cumplió ni se cumple de manera acabada, allí donde supuestamente el principio está o estaba vigente. Identificamos cinco tipos de situaciones en las que algunos paquetes de información reciben tratamientos discriminatorios: gestión del tráfico, redes de distribución de contenidos, priorización por parte de buscadores, discriminación geográfica y diferencia entre la velocidad de carga y descarga. Finalmente, la cuarta sección presenta las conclusiones del trabajo y plantea algunos interrogantes respecto a las políticas públicas en la materia, así como futuras líneas de investigación. 


\section{Definiciones, discursos y actores}

\subsection{Antecedentes del concepto de NN}

Una posible genealogía desde las regulaciones estatales ubica los antecedentes del debate sobre la "neutralidad de las redes" en las disputas por el "acceso abierto" ocurridas a finales de los noventa en los Estados Unidos (McChesney, 2013; Newman, 2016). El acceso abierto se refería en este contexto a la posibilidad de que compañías proveedoras de servicios de internet pudieran utilizar la infraestructura de las redes de las firmas de telecomunicaciones y cable a precios no discriminatorios. Tras diversas resoluciones parciales, la Comisión Federal de Comunicaciones (FCC) rechazó el requisito del acceso abierto tanto a las redes de telefonía como a las de cable.

En ese contexto es que Tim Wu introduce la expresión "neutralidad de la red" por primera vez en su texto Network neutrality, broadband discrimination (2003). Se trataba de discutir acerca del rol potencialmente arbitrario y discriminatorio de los proveedores de servicios de banda ancha en las prácticas de transmisión, conmutación y enrutamiento de los paquetes de datos. En 2005, haciéndose eco de los discursos e intereses afines a la perspectiva de Wu, la FCC adoptó una serie de principios que apuntaban a que las redes de banda ancha fueran "accesibles" y "abiertas" . A su vez, en 2010, el organismo implementó una serie de reglas (Open Internet Report and Order, 2010) de "no bloqueo" y "no discriminación" por parte de prestadores de banda ancha, sujetas a excepciones justificadas en virtud de una "razonable" administración de las redes. Estas medidas fueron profundizadas en 2015, durante la Presidencia de Barack Obama en los Estados Unidos, cuando se promulgaron las llamadas Open Internet Rules (FCC, 2015).

2. Estos principios, conocidos como "las cuatro libertades de Internet", procuraban asegurar: a) el derecho de los consumidores a acceder a cualquier tipo de contenido legal; b) la libertad para ejecutar aplicaciones y servicios; c) la libertad para conectar cualquier dispositivo legal que no dañe la red; d) la libre competencia entre proveedores de redes, proveedores de aplicaciones y servicios, y proveedores de contenidos (FCC, 2005). 
Estas reglas establecieron tres principios básicos para todos los proveedores de servicios de banda ancha fijos y móviles: a) no bloqueo en el acceso a contenido legal, aplicaciones, servicios o dispositivos no dañinos; b) no estrangulamiento (throttling) o degradación del tráfico lícito en internet, y c) no prioridad por pago, es decir, que no existan "carriles rápidos" para favorecer cierto tráfico en detrimento de otro, a partir de "intercambios" o arreglos comerciales.

El panorama regulatorio volvió a cambiar con la llegada de Donald Trump al gobierno y la decisión de revisar las reglas sobre neutralidad de la red. Así, en junio de 2018 se derogaron las Open Internet Rules de 2015, con el argumento de que la regulación habría afectado negativamente a las inversiones en el sector de infraestructura de las telecomunicaciones.

Desde entonces, diversos actores alineados con los discursos e intereses favorables a la NN han manifestado su oposición a los cambios (entre ellos, las empresas de Silicon Valley y los pioneros de internet, la WWW y las tecnologías digitales como Vinton Cerf, Tim Berners Lee y Steve Wozniak). Actualmente existen algunas iniciativas legales en ciertos estados (Washington, Oregon, California) y a nivel nacional (por parte del Partido Demócrata) para restaurar las reglas de $2015^{3}$, aunque su horizonte es incierto.

\subsection{Discursos y actores}

En este trabajo entendemos que existen dos tipos de definiciones de neutralidad de la red ${ }^{4}$ y dos grupos de actores cuyos intereses expresados discursivamente vertebran el debate. Asimismo, sugerimos que uno de estos grupos realiza una operación retórica crucial, que no siempre se advierte, combinando ambos tipos de definiciones.

3. Véase "Democrats unveil bill to restore US 'net neutrality", 6 de marzo de 2019. Disponible en https:// phys.org/news/2019-03-democrats-unveil-bill-net-neutrality.html

4. Naturalmente, no todas las definiciones pueden reducirse a uno de estos tipos ideales. Se trata, apenas, de una simplificación que nos permite entender operaciones discursivas usuales. 
El primer tipo corresponde a las definiciones que aquí denominamos "restringidas", es decir, aquellas que se ciñen a aspectos económicos y técnicos. Entre estas ${ }^{5}$ encontramos la siguiente: "Entendemos a la neutralidad de la red como una restricción en la diferenciación de precios o discriminación de precios por parte de las empresas involucradas en la cadena de valor de los servicios de comunicaciones, particularmente aquellas que brindan transporte" [traducción propia] (Cave \& Crocioni, 2007: 670).

El segundo tipo se asocia con definiciones más amplias, que incluyen elementos culturales y axiológicos. Resulta representativa la síntesis siguiente: "La idea rectora es que todo tráfico de datos debe ser tratado en condiciones de igualdad, con el propósito de garantizar a los usuarios igual libertad de elección respecto a los contenidos que quieran consumir, transmitir o crear" (Fontanals, 2017: 1) ${ }^{6}$. En este segundo tipo de definiciones, el énfasis en la noción de neutralidad de la red aparece estrechamente ligada a principios como igualdad, libertad de elección y rechazo a la discriminación, es decir, conceptos relevantes para la teoría política en general y especialmente asociados al orden democrático.

5. Definiciones similares se hallan en Baumol et al. (2007: 1): "La neutralidad de la red es una propuesta de política pública que, entre otras cosas, regularía cómo los proveedores de red administran el uso de sus redes y cuál sería su precio" [traducción propia]; y en Hahn y Wallsten (2006: 1): "La neutralidad de la red no posee una definición precisa ampliamente aceptada, pero generalmente significa que los proveedores de servicios de banda ancha cobran a los consumidores solo una vez por el acceso a Internet, no favorecen a un proveedor de contenido sobre otro, y no les cobran a los proveedores de contenido por el envío de información a los usuarios finales a través de la banda ancha. En otras palabras, 'neutralidad de la red' es, en realidad, un nombre amigable para la regulación de precios" [traducción propia].

6. En el mismo sentido: "La defensa en pro del principio de neutralidad de red es también en pro de la oportunidad de emancipación, individual, colectiva y considerada igualmente entre todos los del globo, con la finalidad de dignificar al hombre y todos los hombres, en un espíritu de fraternidad... Decimos esto, pues la oportunidad de emancipación individual y colectiva es inmediata gracias a la difusión constante y permanente de información y conocimiento, permitida y producida a través de las plataformas de comunicación, mayoritariamente de Internet" (Lopes Matsushita, 2014: 16). O también: "Así, se puede inferir que el concepto se encuentra atravesado por dos compromisos de no discriminación diferentes: el del servicio universal -relacionado con el acceso igualitario a todos los individuos- $\mathrm{y}$ otro de servicio público de transporte -o common carriage, que contempla el trato igualitario de todos los contenidos que circulan por la web, sin diferenciarlos por sus costos, peso, tipo u origen” (Fernández, 2014: 71). 
En cuanto a los actores interesados y sus discursos, el grueso del debate acerca de la neutralidad de la red se encuentra vertebrado por dos posturas que responden a posiciones de sendos tipos de actores e intereses. Una primera postura argumenta a favor de la neutralidad de la red que cualquier discriminación indebida por parte de los proveedores de conectividad en relación a los contenidos legítimos, aplicaciones o servicios a los que los usuarios desean acceder supone una amenaza para la competencia, para la innovación (situada en los extremos de la red) y para el ejercicio de derechos fundamentales como la libertad de expresión. Este enfoque, que promueve la aplicación de reglas de neutralidad, es impulsado por los grandes proveedores de contenidos y servicios y por diversas organizaciones de la sociedad civil que trabajan sobre derechos digitales (Global Net Neutrality Coalition, s/f; Google Inc., 2010; Hastings, 2014; Internet Association, 2017; Intervozes \& Derechos Digitales, 2017). Una segunda posición, avalada por los grandes operadores de redes y contraria a la $N N$, sostiene que la cantidad abrumadora de datos que circula por internet debe ser gestionada, organizada y catalogada según el ancho de banda que esta requiera y que las costosas inversiones necesarias para la expansión de las redes suponen que las empresas puedan asegurarse rendimientos económicos razonables. Ello, a su vez, estimularía una mayor inversión e innovación en el ecosistema total de internet (Attwood, 2011; Lambert, 2010; Medina, 2017; Pepper \& Maz, 2011; Schneibel \& Farivar, 2010) (ver Tabla I).

\section{Tabla I}

Actores y posiciones sobre la regulación de la neutralidad de la red

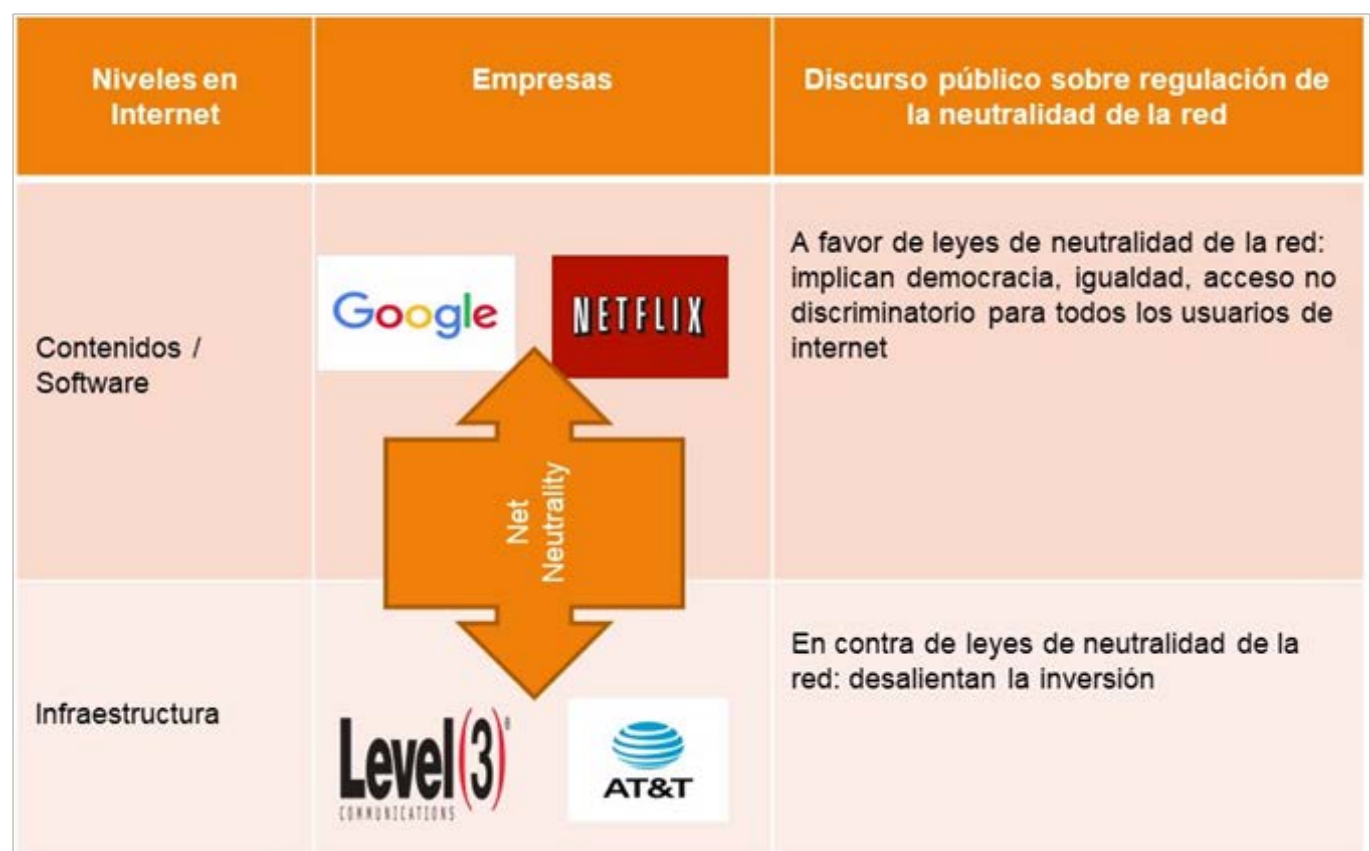

Fuente: elaboración propia 
La primera posición es, como se señaló, la que concita mayores adhesiones. A modo ilustrativo, en los medios de comunicación masiva, una búsqueda rápida en los diarios argentinos La Nación y Clarín sobre "neutralidad de la red" arroja como resultado unas 241 noticias en el primer caso y 315 en el segundo. Simplificadamente puede decirse que en todas ellas la noción de "neutralidad de la red" (especialmente tal y como se presenta en el segundo tipo de definición) es aceptada sin mayores reservas, de manera tácita o explícita, como válida, conveniente y justa. Así, la mayoría de los artículos periodísticos se refieren a las discusiones y reglas que rigen los Estados Unidos y Europa ${ }^{7}$, retomando los principios hegemónicos asociados al debate: neutralidad de la red vinculada con la libertad de expresión, la igualdad y la democracia ${ }^{8}$ y con la idea de "una internet libre, abierta e inclusiva" 9 . Si bien aparecen notas de opinión que tratan de examinar diferentes perspectivas, en general no cuestionan la existencia misma de la neutralidad de la red ni su puesta en práctica ${ }^{10}$.

Pero no se trata solo de que haya un tipo de discurso dominante asociado a los intereses de determinados actores. El punto central consiste en la forma que asumen estos discursos. En efecto, los discursos sobre la neutralidad de la red impulsados por los proveedores de servicios y contenidos y replicados por una amplia gama de actores sociales utilizan una retórica asociada al segundo tipo de definición, esto es, una retórica vinculada a la igualdad, la libertad y la democracia. Sin embargo, en la práctica (legislativa y mediática) se rechazan más bien (o exclusivamente) las discriminaciones de paquetes que violan la primera definición, esto es, la discriminación de precios por parte de los proveedores de conexión y los ISPs. Como señalan Cave y Crocioni:

7. Cuando se refieren a la Argentina suelen enfocarse en cómo afectarían localmente los cambios regulatorios de los EEUU (véase, por ejemplo: https://bit.ly/2Iw1obN o https://bit.ly/2Gd9010) o en alguna modificación normativa bajo tratamiento en el Congreso de la Nación (véase en relación al supuesto proyecto de "ley de comunicaciones convergentes" el siguiente enlace https://bit.ly/2Z4lPCb).

8. Véase, por ejemplo: https://bit.ly/2P0FrTa o https://bit.ly/2D5wDGY

9. Véase, por ejemplo: https://bit.ly/2KoD8uz

10. Véase una excepción interesante: https://bit.ly/2Z4muUb 


\begin{abstract}
Hay un aspecto de economía política en el debate de la neutralidad de la red. Una caracterización aproximada, pero que creemos suficientemente precisa, es que la neutralidad de la red es un intento de los proveedores de contenido y aplicaciones -del tipo de Amazon, eBay, Google, Microsoft, Yahoo! e Intel- para restringir el comportamiento de los proveedores de acceso a Internet de banda ancha - tales como AT\&T, Verizon, Comcast y Sprint- a través de la presión política [traducción propia]. (Cave \& Crocioni, 2007: 670)
\end{abstract}

Así, los proveedores de servicios y contenidos suelen hablar en nombre de los intereses de la totalidad de los actores del ecosistema de internet e incluso de valores que se extienden a las sociedades democráticas en general. Aun así, en la práctica defienden intereses particulares considerando como violaciones a la neutralidad de la red solo aquellas actividades contrarias a sus negocios. Un ejemplo simple y no por ello menos relevante de este tipo de operación puede encontrarse en un comunicado de Google a sus usuarios, firmado por su presidente ejecutivo Eric Schmidt en 2006:

\begin{abstract}
Internet, como la conocemos, se enfrenta a una seria amenaza. Hay un debate que está subiendo de tono en Washington D.C., sobre algo que se denomina 'neutralidad de la red', y es un debate tan importante que Google está pidiendo que se involucren. Les pedimos que tomen medidas para proteger la libertad en Internet. [...] Hoy en día, Internet es una autopista de la información en la que cualquier persona -sin importar cuán grande o pequeña sea, cuán tradicional o no convencional-, tiene un acceso igualitario. Pero los monopolios de telefonía y cable, que controlan casi todo el acceso a Internet, quieren poder elegir quién tiene acceso a los carriles de alta velocidad, y qué contenido se ve primero y más rápido. Quieren construir un sistema de dos niveles de velocidad y bloquear los carriles de acceso para quienes no puedan pagar [traducción propia, énfasis añadido]. (Donham, 2010)
\end{abstract}

La cita de Google (al igual que muchas otras) presenta la idea de que cualquiera tiene un acceso similar a internet. Esta declaración tiene fuertes connotaciones políticas y en ella resuenan principios democráticos fundamentales. Sin embargo, no resulta claro que la neutralidad de la red, entendida de ese modo y en un sentido estricto, haya existido ni en un período originario de la internet comercial ni en la actualidad, allí donde existen regulaciones que discursivamente buscan preservar tal neutralidad. En la siguiente sección intentaremos argumentar en favor de esta afirmación. 
Por supuesto, no se trata de una curiosidad discursiva, sino de señalar que empresas como Google (y otras similares, junto con sus ONG satelitales) aceptan sin mayores miramientos e incluso silencian las alteraciones de la neutralidad de la red (de acuerdo a su propia definición y discursos), mientras estas no afecten a su esquema de negocio. En cambio, identifican y movilizan toda clase de recursos para detener aquellas violaciones a la neutralidad de la red que inciden en su estructura comercial. En ciencias sociales, uno de los conceptos usuales para dar cuenta de este mecanismo es el de ideología. Sin embargo, carece de utilidad si es asociado a la idea de una "falsa conciencia". Lo que interesa aquí es pensar la noción de ideología como herramienta para explicitar los fundamentos ocultos de los discursos, es decir, para mostrar que no se condicen con la pretendida racionalidad de su enunciación, y que, si bien constituyen verdades parciales, buscan ocultar los condicionamientos materiales de los actores que los enuncian y las disputas de poder que los conforman ${ }^{11}$. Es en este sentido que los discursos que analizamos aquí, y especialmente el de los actores del nivel de la provisión de contenidos y servicios que bregan a favor de la NN, pueden ser caracterizados como ideológicos.

\section{Del discurso a la práctica}

En la literatura revisada y en las conversaciones con especialistas han emergido señalamientos reiterados respecto de que en la práctica hay diversas situaciones en las que se produce un tratamiento dispar de los paquetes de información. Aún en contextos en los que la neutralidad de la red está consagrada legalmente, se avista una variedad de prácticas difícilmente compatibles con una noción profundamente democrática de la neutralidad de la red. Sin embargo, hasta donde tenemos noticias, no se ha producido una sistematización de esas situaciones en la literatura académica. Es por eso que el aporte principal de este trabajo consiste en intentar una sistematización, que posiblemente sea limitada y no exhaustiva. Así, hemos identificado cinco tipos de situaciones (distintas de las denunciadas por los discursos pro neutralidad de la red) en las que se advierte que los paquetes, y los usuarios que los envían, no son tratados de manera neutral.

11. Los autores como Zizek (2003) que proponen esta noción específica de ideología lo hacen combinando a Marx con el psicoanálisis (García \& Aguilar Sánchez, 2008). 


\subsection{Gestión del tráfico}

En primer lugar, las técnicas de gestión del tráfico, que son contrarias al principio de neutralidad de la red, son de aplicación usual. Para comprender este tipo de prácticas conviene partir del funcionamiento del principio de conmutación de paquetes (packet switching) sobre el cual se basa la transmisión de información entre redes de computadoras en todo el mundo. Este supone que cada mensaje que se envía sea "troceado" en paquetes de datos, "enrutado" a través de la red, etiquetado con un número de secuencia y dirección de destino. Cada enrutador (router) envía los paquetes de datos en función del orden en que han sido recibidos, a través de la ruta más rápida. Por lo tanto, ciertos paquetes "viajan" con prioridad de paso sobre otros. Así, existe un trato preferencial de algunos paquetes que suele ser aludido como una "discriminación natural del tráfico" (Fontanals, 2017), en la cual se prioriza el tránsito de ciertos paquetes de datos sobre otros.

Esta situación se relaciona con el paradigma end-to-end en el que se basa el diseño de internet, y que ofrece las ventajas de una red abierta pero las desventajas de la congestión y la fluctuación. Si bien nos permite comunicarnos en instantes con alguien localizado en el otro extremo del planeta, cuanto mayor sea la distancia física entre la fuente y el destino, mayor será el número de nodos que deberá atravesar y mayores las probabilidades de congestión, con las consiguientes pérdidas de paquetes de datos o retardos. 
Con el argumento de evitar la congestión de las redes en determinados momentos en los que el tráfico aumenta, los proveedores de servicios de internet recurren al uso de técnicas de "gestión del tráfico", también conocidas como traffic prioritisation, traffic shaping o access-tiering (Georgieva, 2014: 49). En dicha tarea, los ISP pueden retrasar ciertos paquetes de datos respecto de otros, ayudados por tecnologías como la "inspección profunda de paquetes" (deep packet inspection, DPI). Las técnicas de DPI introducen "inteligencia" en lo que en general se ha denominado una red "tonta" (dumb network), lo que facilita no solo la discriminación de los paquetes de datos sino también una "vigilancia integral" puesto que permite a los ISP monitorear, acelerar, frenar, bloquear o filtrar determinado tráfico (Bendrath \& Mueller, 2011).

Los ISP tienen la posibilidad de ver el contenido de un paquete de datos y saber, por ejemplo, a través de distintos indicadores, si precisa de un transporte de alta velocidad (una comunicación de telefonía por VoIP o transmisión de vídeo) o si requiere de un ancho de banda menor. Sin embargo, ello también los habilita a priorizar tráfico para quienes paguen por ello, limitar cierto tráfico que les genere costos (por ejemplo, descargas P2P), promover cierto contenido de empresas afiliadas en el caso de los ISP integrados verticalmente, o bloquear los servicios ofrecidos por la competencia.

En efecto, las prácticas de gestión del tráfico son habituales incluso en países que cuentan con regulaciones sobre neutralidad de la red. Así, los operadores de redes distinguen entre tipos de tráfico y priorizan su tránsito por motivos que el usuario desconoce, lo que contraía el principio de "extremo a extremo". En el caso de la Argentina, expertos de la Fundación Sadosky y empresarios del sector ${ }^{12}$ nos confirmaron que las prácticas de gestión de tráfico son usuales en el país.

12. En ambos casos, nuestros entrevistados prefirieron permanecer anónimos. Las consultas fueron realizadas en abril de 2018. 


\subsection{Redes de distribución de contenidos (CDN)}

En segundo lugar, determinados usos de la noción de neutralidad de la red invisibilizan el hecho de que existen proveedores de contenidos o servicios de diferentes tamaños y grados de poder económico. En este sentido, los gigantes de internet pueden recurrir a redes de distribución de contenidos (CDN, content delivery network) que contienen copias de datos colocados en varios puntos de la red. Ello permite a sus clientes eludir las rutas congestionadas y acceder a copias del contenido descargado con mayor frecuencia más cerca de la red de acceso del cliente, en lugar de ir al servidor central, a fin de maximizar el ancho de banda, mejorar el tránsito de los servicios y la experiencia de los usuarios. En varios casos, las empresas suelen suscribir acuerdos de interconexión (peering) con los operadores de redes, lo que les permite conectar sus servicios directamente a las redes de los ISP. De esta forma, los contenidos viajan directamente desde un determinado proveedor al operador, minimizando el paso a través de redes de terceros. En otras palabras, las CDN permiten que los paquetes de información de quienes las utilizan lleguen más rápido que los paquetes de otras empresas o usuarios.

Esta práctica es muy común entre los grandes operadores de contenidos, tales como Facebook, Netflix y Google. Netflix, por ejemplo, lanzó su propia red de distribución de contenidos llamada "Open Connect" en 2011, para dejar de depender de las clásicas CDN de terceros (Akamai, Limelight y Level 3), capaces de soportar el ancho de banda requerido por el tráfico de esta empresa ${ }^{13}$. Netflix ha suscrito cientos de acuerdos de interconexión con redes privadas y públicas en todo el mundo. Una investigación de la Queen Mary University of London

13. El programa se maneja con dos componentes principales: los "dispositivos integrados" (open connect appliances, OCA) y la "interconexión sin compensación” (settlement-free interconnection, SFI). En el primer caso, los dispositivos OCA se incorporan a la red de los ISP asociados, donde almacenan los contenidos de la plataforma para distribuirlos hacia los usuarios lo más rápido posible, sin necesidad de acudir al servidor central. En el segundo, los dispositivos son instalados en diferentes centros de datos y "puntos de intercambio de internet" (internet exchange points, IXP) en mercados relevantes para la empresa, desde donde se interconectan con los ISP a través de acuerdos de peering. Fuente: https://openconnect.netflix. com/es_mx/ 
encontró que en 2016 Netflix contaba con 4669 servidores que distribuían su contenido por internet en 243 ciudades del mundo, reduciendo sensiblemente el trayecto que debe recorrer la información en comparación con los proveedores de contenido que parten de servidores centralizados (ver Figura 1).

\section{Figura 1}

CDN de Netflix

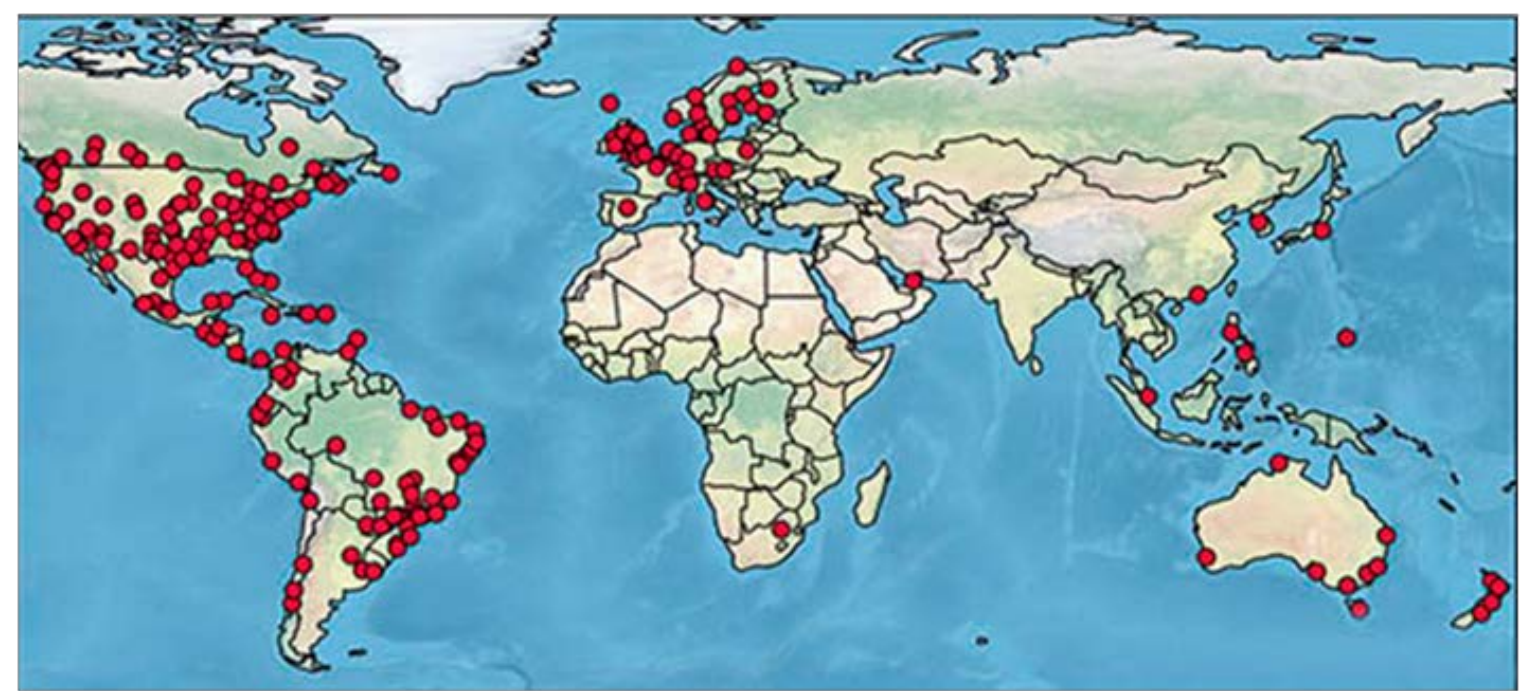

Fuente: Böttger, Cuadrado, Tyson, Castro, \& Uhlig (2018)

Facebook también anunció el lanzamiento de su propia CDN, apenas después que Netflix, mientras continúa utilizando redes de distribución de contenidos de terceros, principalmente la de Akamai. Google, por su parte, no solo tiene acuerdos con Akamai y otros proveedores de redes de distribución (Cloudflare, Fastly, Level 3), sino que también ha desarrollado una red de centros de datos (hasta ahora posee nueve en América, cuatro en Europa y dos en Asia), ha establecido cientos de acuerdos de interconexión a través de sus "puntos de presencia" (edge points of presence, PoPs) en todo el mundo, y desarrollado un despliegue de nodos de borde (Google Global Cache, GGC) para que los contenidos se entreguen lo más cerca posible del usuario final ${ }^{14}$.

14. Véase: https://peering.google.com/\#/infrastructure 
Como señalan Krämer et al. (2013), el hecho de que estos proveedores se preocupen por mejorar la calidad de la experiencia de los usuarios está directamente relacionado con su modelo de negocio, toda vez que sus ingresos están vinculados con el valor y el rendimiento del contenido o los servicios ofrecidos, así como con la cantidad de usuarios, lo que reporta mayores ingresos por anuncios publicitarios. Si los consumidores experimentan una mala calidad del servicio debido a la congestión en la red, podrían asociar las dilaciones con el sitio y, eventualmente, darlo de baja.

Las CDN han despertado intercambios entre los bandos enfrentados acerca de la neutralidad de la red. Los operadores de redes suelen afirmar que las CDN no son neutrales, puesto que permiten a los proveedores de aplicaciones y contenidos tener una situación de ventaja competitiva frente a sus rivales en la batalla por brindarles a los usuarios finales experiencias de internet de alta calidad, lo que marca "un cambio de poder sin precedentes dentro del ecosistema de Internet" (AT\&T, 2010). Por su parte, estas empresas responden que la función de las redes privadas es mejorar la experiencia general en el uso de internet, alojando y sirviendo contenidos desde una ubicación más próxima a los usuarios finales, evitando puntos de posible congestión y reduciendo la latencia. Sostienen que no interfieren con otros flujos de tráfico hacia los usuarios finales, cuyo control queda en manos de los proveedores de acceso de banda ancha de última milla (Google Inc., 2010). Según este argumento, ello no iría en contra del principio de neutralidad de la red porque la mejora en el rendimiento de determinado sitio no se ha desarrollado en detrimento de ningún otro, y los desarrollos estarían disponibles para todos los demás (Misener, 2011).

Sin embargo, en la mayoría de los debates sobre neutralidad de la red no se suelen discutir las redes de distribución de contenidos ni los acuerdos de interconexión, pese a que favorecen el tráfico de determinados operadores dejando al resto en una situación de desventaja. Ello contradice no solo la idea de la libre competencia en la capa de contenidos, sino también el principio de que el 
tráfico de datos viaja en condiciones de igualdad.

El desarrollo de CDN por parte de determinadas firmas, claro está, implica que los contenidos que cuenten con mayor inversión de capital viajarán más rápido. El hecho de que una parte de su trayecto ocurra por fuera de lo que se conoce como internet (y esto es lo que genera la falsa idea de que no afecta los principios enunciados por la neutralidad de la red) es un fenómeno secundario. Lo que está en juego es si las redes de información digital ponen a los distintos actores en pie de igualdad o si, por el contrario, ejercen una forma de discriminación. En este caso, como en otros, se advierte que hay un tipo específico de paquetes que en la totalidad del proceso de transmisión resultan perjudicados: los que son inyectados en la red por productores con menor poder económico. En este sentido, el devenir de la dinámica económica de las CDN no es difícil de imaginar: todas las empresas proveedoras de servicios y contenidos desarrollan sus CDN, mientras que las pequeñas empresas, los "prosumidores" e incluso los actores estatales deben conformarse con el hecho de que sus contenidos circulen de manera más lenta o realizar acuerdos con los ISP pagándoles adicionalmente para que prioricen sus contenidos a fin de poder competir con los que circulan a través de las CDN.

Por supuesto, se advierte que ambas modalidades son contrarias a los principios de la neutralidad de la red (y no solo a ellos, sino a los valores asociados a una internet democrática). Sin embargo, los debates actuales en defensa de la libertad y la igualdad tienden a llamar la atención solo sobre los segundos (los acuerdos entre determinados proveedores, los "chicos" en nuestro caso hipotético, y los ISP), mientras que los primeros (las CDN conectadas directamente a los IXP o con acuerdos con los ISP) son entendidos dentro de las normas de juego de la neutralidad de la red. 


\subsection{Priorización por parte de buscadores}

En tercer lugar, la priorización de algunos paquetes de información sobre otros que ejercen los buscadores en internet, por medio de opciones de pago y gratuitas, también va en contra de los discursos pro neutralidad de la red (Cave \& Crocioni, 2007: 671).

Aunque resulte contra intuitivo - debido a los discursos impulsados por los proveedores de contenidos y servicios y actores afines-, es fundamental preguntarse acerca de la discriminación sobre los paquetes de datos que ejercen los buscadores. Si la discriminación de paquetes en el nivel de la infraestructura, por ejemplo en función de un pago adicional, es contraria al principio de neutralidad, ¿no lo sería la priorización de paquetes en el nivel del software, especialmente en casos de cuasi monopolio? Este tipo de preguntas, así como el término search neutrality, emergen en el trabajo de Odlyzko (2009).

En el año 2009, Adam Raff, cofundador de Foundem (un sitio que realiza "búsquedas verticales" en la web para comparar productos online en el mercado británico), denunció a Google por lo que juzgaba eran penalizaciones aplicadas por el motor de búsqueda que redundaron en una disminución del tráfico del sitio. El debate giró en torno a si el modo en que operaba Foundem, considerado por algunos como una forma de spam, podía ser castigado por un buscador. La Comisión Federal de Comercio de los Estados Unidos investigó si Google había manipulado sus algoritmos para dañar sitios web verticales y promover injustamente sus propias empresas verticalmente integradas, aplicando un "sesgo de búsqueda" (Federal Trade Commission, 2013). Sin embargo, cerró la investigación determinando que no violaba la legislación en materia de defensa 
de la competencia.

La analogía entre la llamada net neutrality y la search neutrality ha sido sistemáticamente indagada por Georgieva (2014), quien concluye que los mismos principios que sostienen la primera conllevan a impulsar la segunda. Sin embargo, la autora constata que en el presente hay una serie de asimetrías, de limitaciones al tratamiento neutral de los paquetes de información en virtud del funcionamiento técnico, regulatorio y comercial de los buscadores.

En primer lugar, los buscadores - al igual que los ISP - funcionan como information gatekeepers, teniendo la posibilidad de excluir en términos prácticos el acceso a determinados dominios (Grimmelmann, 2013; Manne \& Wright, 2011; Zelnick \& Zelnick, 2013). En segundo lugar, estas empresas también operan gestionando el tráfico (en un sentido similar al señalado en el epígrafe 3.1.) en tanto práctica usual de los operadores de redes. Así lo señala Innocenzo Genna, quien estuviera a la cabeza de la ECTA (European Competitive Telecommunications Association):

El mercado de búsqueda en línea está dominado por un jugador, Google, que potencialmente tiene la capacidad de transportar el tráfico web, tal como un ISP dominante puede hacer con el tráfico de internet. El momento en que Google comienza a privilegiar un destino, en lugar de otro, representa una amenaza para la 'neutralidad' de la búsqueda en línea [traducción propia]. (Genna, 2014: 7) 
En tercer lugar, los buscadores tienen una práctica reñida con la neutralidad que viene dada por el carácter secreto de sus algoritmos. Al igual que la opacidad en la actividad de los ISP alimenta sospechas acerca de su neutralidad en el tratamiento de datos, lo mismo ocurre con las fórmulas de rankeo de empresas como Google, que podrían estar manipulando los resultados de búsqueda e indexación con fines comerciales (Georgieva, 2014: 60). Finalmente, la autora señala cuatro principios que, en aras de un tratamiento neutral de los paquetes de datos, deberían discutirse respecto de los buscadores: transparencia, no bloqueo, no discriminación y gestión razonable.

\subsection{Discriminación geográfica}

El principio que postula que todos los paquetes de datos que circulan por internet deberían recibir el mismo trato y no ser discriminados por su origen, uso o aplicación, tampoco se cumple si tenemos en cuenta las diferencias en términos de velocidad de conexión entre países.

Las velocidades de descarga o bajada describen la velocidad a la que se transfieren datos de internet a la computadora de un usuario, mientras que las velocidades de subida o carga se refieren a la tasa de transferencia de datos en la dirección opuesta, de la computadora de un usuario hacia la red. Ambas velocidades de conexión son menores en contextos periféricos, lo que conlleva que el tráfico de datos desde y hacia dichos países viaje necesariamente más lento, en comparación con aquellos que poseen mejores velocidades de conexión. 
La velocidad de descarga promedio a nivel mundial en redes por banda ancha fija es de 45,07 Mbps (datos de Speedtest.net, de abril de 2018). Singapur encabeza el ranking de países en velocidad de conexión casi cuadriplicando el promedio mundial (174,94 Mbps). Lo siguen Islandia, Hong Kong y Corea del Sur. En séptimo lugar se ubica Suecia con 93,69 Mbps y en el noveno, los Estados Unidos con 91,46 Mbps.

El primer país latinoamericano que aparece en el ranking es Chile, en la ubicación 39, seguido por la República de Trinidad y Tobago, Uruguay y Puerto Rico en los puestos 45, 48 y 50 respectivamente. Argentina aparece en la posición 82 con $18,08 \mathrm{Mbps}$, es decir, por debajo de la mitad del promedio mundial. Varios países africanos y de oriente medio (Algeria, Líbano, Afganistan, Egipto, Libia), junto con algunos latinoamericanos (Venezuela, Bolivia, Nicaragua, Paraguay, Honduras) se encuentran entre los que poseen las peores conexiones (entre 7,32 y 3,73 Mbps) en todo el mundo (ver Tabla II). En todos los casos se trata de velocidades promedio, teniendo en cuenta que los proveedores de banda ancha suelen promocionar la velocidad máxima de descarga de conexión que ofrecen, mientras que la que reciben los usuarios es generalmente menor.

Si bien en la mayoría de los países no existen normas que regulen de manera estricta sobre la neutralidad de la red. En el caso de que las hubiera, estas solo garantizarían que los paquetes de datos desde y hacia los países periféricos viajasen a una velocidad uniformemente lenta, mientras que la de los países centrales lo hicieran a una uniformemente rápida. En otras palabras, los paquetes de datos son discriminados por su locación de procedencia. Por supuesto que esto no se debe a un fenómeno geográfico, sino a las desigualdades económicas que resultan en menores niveles de inversión para las regiones y países en los que la demanda efectiva es menor. Así, es inevitable encontrar diferencias dentro de cada país. La heterogeneidad internacional también se verifica al interior de los ámbitos nacionales, pero el principio es el mismo: los paquetes que provienen de zonas con mayor poder adquisitivo tienen prioridad respecto de los que envían las personas, colectivos, ciudades o países con menores niveles de acumulación de capital. 


\subsection{Diferencia entre velocidad de carga y descarga}

Más allá de las diferentes velocidades entre geografías, también se observa usualmente una discriminación entre los paquetes que se descargan (download) y aquellos que se cargan (upload), de modo tal que los primeros circulan a una velocidad significativamente mayor que los segundos. Como afirman Cave y Crocioni (2007), la diferencia entre velocidades de subida y descarga que ofrecen los proveedores implican la priorización de unos paquetes sobre otros.

Así, mientras que el promedio mundial de velocidad de descarga es de 45,07 Mbps, el de carga es de 21,93 Mbps, es decir, un 51,3\% más lento. Esta diferencia, previsiblemente, disminuye en algunos países y se incrementa en otros. La dispersión por países es significativa, pero para los fines de este breve trabajo, los promedios por continente ofrecen resultados suficientes (ver la Tabla III).

La tabla muestra con claridad que en todos los continentes las velocidades promedio de carga son bastante menores que las de descarga ${ }^{15}$. Esto no debe opacar algunas diferencias notables que existen entre las distintas regiones. En términos de los valores absolutos de la velocidad de carga, África y, especialmente, América Latina y el Caribe presentan valores bastante por debajo del promedio mundial. Sin embargo, en términos de la diferencia porcentual entre ambas velocidades, es de destacar que América del Norte (Estados Unidos y Canadá) presenta el valor más extremo, aunque América Latina y el Caribe se hallan apenas por detrás. Resulta valiosa la comparación de esta región con África: mientras América Latina y el Caribe presentan una velocidad de descarga mayor que la de África, la de carga es menor. Nuestro continente se caracteriza, también en este aspecto, por la combinación de limitaciones y asimetrías. Por supuesto, hay asimismo desigualdades entre los países reducidos a un promedio.

15. En términos de países individuales hay varias excepciones. En algunos casos se trata de países con altas velocidades de conexión, tales como Singapur, con una tasa de carga de 185,45 Mbps frente a una de descarga de 174,94 Mbps. 
Siendo el continente en el que se encuentra el país en el que se origina esta investigación y hacia el que irán destinadas nuestras eventuales sugerencias de políticas públicas, presentamos una desagregación de los valores para cada país de la región de América Latina y el Caribe (ver Tabla IV). Con las excepciones parciales de Ecuador y Belice, todos los países de la región presentan enormes diferencias porcentuales entre las velocidades de carga y descarga de contenidos. Los casos más extremos son los de Uruguay, Chile, Puerto Rico, Panamá, Perú, Guam, Argentina y República Dominicana. En ellos, la diferencia entre las velocidades de carga y descarga es mayor al 70\%. Pero esto no debe llevar a asimilar las situaciones de estos países, en tanto que la velocidad de carga en términos absolutos diverge entre ellos. Por caso, la de Uruguay casi triplica la de Argentina. En este sentido, la situación de la Argentina es llamativa y preocupante: sus valores de carga son muy inferiores a los de los países con los que suele compararse en otros indicadores socioeconómicos (Brasil, Chile, Uruguay, México, Colombia) y se asemejan a los que presentan países con productos per cápita muy inferiores.

Ahora bien, retomando la Tabla II, el hecho de que los valores de carga sean menores que los de descarga en todos los promedios continentales podría inducir a pensar que esta discriminación de paquetes obedece a razones técnicas difícilmente salvables. Sin embargo, hay varios casos de países en los que se verifica la situación inversa, como ilustra la Tabla V. Se observa cómo una velocidad de carga similar o superior a la de descarga no solo se encuentra en los casos de países que cuentan con velocidades altas (Japón, Singapur e Islandia), sino también en otros con velocidades intermedias o bajas. Asimismo, se trata de países situados en distintos continentes y con grados de desarrollo económico diverso. Por supuesto, estas situaciones 
podrían explicarse, en algunos casos, por razones técnicas. No obstante, hay un componente de decisión política y regulatoria que resulta fundamental a la hora de definir el perfil del vínculo entre las naciones e internet.

Esto es especialmente relevante en relación a la preocupación que manifiestan algunos partidarios de la neutralidad o apertura de la red respecto de las posibilidades de los emisores de menor capacidad económica (Bernstein \& Pickard, 2017; Cave \& Crocioni, 2007). En efecto, si bien las velocidades de carga no suelen ser una prioridad para el usuario residencial, tienen incidencia sobre las posibilidades de subir archivos, fotos y vídeos, y realizar llamadas por VoIP. Las velocidades de carga de contenidos son, de hecho, un elemento central a la hora de pensar una internet horizontal en la que, tendencialmente, todos los receptores puedan ser a la vez emisores. En cambio, las situaciones de mayor asimetría entre velocidades de carga y descarga ejemplifican diseños en los que las divergencias entre usuarios y productores son mayores, y en los que se concibe a internet más como un medio de consumo que como un medio de producción.

\section{Conclusiones}

En este artículo partimos de identificar dos tipos ideales de definición sobre la neutralidad de la red: uno restringido, que la delimita en relación a la inexistencia de discriminaciones de precios y aspectos técnicos por parte de los operadores de redes, y otro más amplio, que la opone a todo tipo de discriminación y desigualdad en el tratamiento de los paquetes que circulan por internet, basado en argumentos vinculados al orden democrático, la igualdad, la libertad de elección y de expresión. 
Uno de los hallazgos de este trabajo consistió en identificar un recurso discursivo de los proveedores de contenidos y servicios en internet, quienes proponen conceptualizaciones acerca de la neutralidad de la red que se corresponden con el segundo tipo de definición, mientras que en sus propias prácticas consideran como violaciones de la NN aquellas que se corresponden con el primer tipo de definición. Así, no conciben como contrarias a la neutralidad de la red aquellas situaciones que lo son, de acuerdo a los principios de la segunda definición que ellos mismos difunden, cuando estas resultan contrarias a sus intereses comerciales $^{16}$ (ver Tabla VI). Es importante señalar que no se trata de que las empresas proveedoras de contenidos y servicios (por ejemplo, Google o Netflix) mientan, sino de que presentan verdades parciales que ocultan lo esencial, y que identificamos más arriba con la noción de ideología.

Este artículo ofrece un argumento simple: aún en contextos de "neutralidad de la red", es decir, en los comienzos de la internet comercial o en aquellos países que cuentan con una regulación específica sobre el tema, no parece verificarse un tratamiento igualitario de todos los paquetes de datos a través de las redes. El aporte principal del trabajo consistió en ofrecer una sistematización de esas limitaciones. Así, mostramos el espesor que puede tener la diferencia entre ambas efiniciones de la neutralidad de la red. Puede y suele suceder que se cumpla formalmente con la ausencia de discriminación de precios por parte de los proveedores de conexión y redes, pero que no haya igualdad en el tratamiento de los paquetes. Así, la neutralidad consagrada puede ser satisfactoria para los proveedores de servicios y contenidos, pero no necesariamente para los usuarios, para las políticas de creación de contenidos locales o para el desarrollo de las regiones periféricas.

16. La otra consecuencia de ese análisis que es apenas mencionada en este trabajo, pero no por ello menos relevante, es que los intereses de los actores provenientes de regiones periféricas no forman parte del debate tal y como se encuentra planteado. 
Específicamente identificamos cinco tipos de situaciones en las que se advierte un tratamiento dispar de los paquetes de información. En primer lugar, aún en países donde existen regulaciones específicas sobre neutralidad de la red, los ISP recurren a técnicas de gestión del tráfico que les permiten priorizar el tránsito de determinados paquetes de datos en detrimento de otros. Si bien esto en muchas ocasiones se encuentra catalogado dentro de las "excepciones" a las reglas de neutralidad con el fin de evitar la congestión de las redes en momentos en los que el tráfico aumenta. La escasa transparencia que caracteriza a estas prácticas y la falta de control permiten a los operadores de redes priorizar tráfico por motivos económicos y/o por acuerdos comerciales.

En segundo lugar, hallamos que las redes de distribución de contenidos (CDN) son una práctica habitual por medio de la cual las grandes empresas de contenidos y servicios suscriben acuerdos de interconexión y alojamiento de datos para que sus contenidos lleguen más rápido a los usuarios finales, evitando su paso por redes de terceros. Ello contradice el principio de que los paquetes de información viajan en condiciones de igualdad puesto que favorece a un determinado tráfico en detrimento de otro, va en contra de la libre competencia en la capa de contenidos y supone una discriminación entre actores de distinto tamaño y poder económico. 
En tercer lugar, retomamos la analogía que plantean algunos autores entre los conceptos de net neutrality y search neutrality, para señalar que la opacidad en la gestión del tráfico por parte de los operadores de redes se asemeja a la opacidad en el manejo de los algoritmos por parte de los buscadores. Estos actores pueden manipular resultados de búsqueda con fines comerciales y así priorizar determinados paquetes de información sobre otros.

En cuarto lugar, desarrollamos cómo las diferencias en términos de velocidad de conexión a internet entre países conllevan una discriminación geográfica. Los paquetes de datos que provienen de países con mayor poder adquisitivo $y$, por ello, mayores velocidades de conexión, poseen prioridad en el tránsito de información respecto a aquellos que tienen menores niveles de acumulación de capital.

Por último, señalamos que la diferencia entre las velocidades de carga y descarga de datos a internet también conlleva una priorización en el tráfico de unos paquetes de datos sobre otros. Ello se agrava en países que poseen velocidades de carga y descarga muy por debajo del promedio mundial, particularmente en las regiones de África y América Latina.

El tratamiento desigual de los paquetes de información debido a razones económicas, en detrimento de los usuarios de menor poder adquisitivo (en su expresión geográfica u otras) usualmente no es señalado por ninguno de los actores del debate como una afrenta a la neutralidad de la red, por más que contraría los principios que ella supone. Es importante señalar que aquí no hay juicio moral alguno respecto de las prácticas que conducen a este resultado limitante de lo que se define como neutralidad de la red. Solo interesa señalar la contradicción entre los discursos acerca de la igualdad que habría en el tratamiento de paquetes de datos y la situación empírica en la que esa igualdad no se verifica. 
La idea de un principio armónico universal se ha utilizado reiteradamente en la historia de la humanidad, y en la del capitalismo en particular, para disimular los diferentes intereses en pugna o, más precisamente, para naturalizar un estado de cosas que favorece a determinados actores en detrimento de otros. En este caso, la operación ideológica se realiza utilizando el término "neutralidad", que de por sí sugiere prescindencia, equivalencia o nivelación del terreno.

¿Cuál es la respuesta en términos de políticas públicas? Este breve artículo no ha tenido como objetivo responder esta pregunta. Sin embargo, podemos señalar inquietudes que sí surgen de la investigación realizada y que apuntan a caminos que pueden resultar fértiles para futuras indagaciones. Por lo pronto, al menos desde la perspectiva de un país de mediano desarrollo como la Argentina, ubicado en el Sur global, no resulta claro que las alternativas reseñadas, esto es, la neutralidad de la red en su versión acotada impulsada por los proveedores de contenidos y servicios y la ausencia de neutralidad propuesta por lo proveedores de conexión y redes, resulten satisfactorias en términos de favorecer el desarrollo de las competencias locales. Por lo tanto, podría resultar valioso reflexionar sobre la posibilidad de concebir otras alternativas, otras modalidades que excedan a las dos posiciones más extendidas. ¿Qué principios deberían guiar esas alternativas? Nuevamente, esa es una materia para futuras indagaciones, pero podemos imaginar que hay espacio para propuestas que pongan en el centro de las preocupaciones políticas a los intereses de actores sociales y los valores asociados al desarrollo - que no son jerarquizados por los bandos en pugna en el debate sobre la NN-: de un lado, los actores sin fines de lucro (es decir, las esferas pública estatal y no estatal), las pymes, las cooperativas, los prosumidores y usuarios; del otro, los valores favorables al impulso de la cultura local, la acumulación y escalamiento de competencias locales, la recuperación de la capacidad de regulación nacional, 
en fin, de aquellos asociados al desarrollo económico y social. Asimismo, uno de los debates cruciales respecto de esas terceras alternativas refiere a cuál es la cantidad y calidad óptima de intervención estatal para nivelar el terreno respecto de las asimetrías que el mercado capitalista (en su versión con neutralidad de la red o sin ella) genera en detrimento de los actores más débiles. Naturalmente, el desarrollo de este debate merece trabajos específicos.

Esta agenda nos conduciría a interrogarnos por las potencialidades, limitaciones y factibilidad de una neutralidad radical, una neutralidad desarrollista, en la cual se garantizara el efectivo acceso igualitario, democrático y universal a internet, entendiendo su vinculación con la creación de oportunidades para el desarrollo económico y cultural. 


\section{Bibliografía}

- AT\&T. (2010). Comments of AT\&T Inc. before the FCC In the Matter of Preserving the Open Internet Broadband Industry Practices (GN Docket No.09-191 WC Docket No. 07-52). Washington DC: Federal Communications Commission.

- ATTWOOD, D. (2011). AT\&T. Innovación en Internet. En J. Pérez Martínez. (Ed.), Neutralidad de red: aportaciones al debate (pp. 143-146). Madrid: Fundación Telefónica - Ariel.

- BAUMOL, W. J., LITAN, R. E., CAVE, M., CRAMTON, P. C., HAHN, R. W., HAZLETT, T. W., ... SAVAGE, S. (2007). Economists' Statement on Network Neutrality Policy. AEI-Brookings Joint Center for Regulatory Studies, RP07-08. doi: 10.2139/ ssrn.976889

- BENDRATH, R., \& MUELLER, M. (2010). The end of the net as we know it? Deep packet inspection and internet governance. New Media \& Society, 13(7), 11421160. doi: $10.1177 / 1461444811398031$

- BERNSTEIN, D. J., \& PICKARD, V. (2017, 22 de diciembre). Why Loss of Net Neutrality Hurts Democracy. Consortium News. Independet Investigate Journalism and Political Review. Recuperado de https://bit.ly/2Db4le4

- BÖTTGER, T., CUADRADO, F., TYSON, G., CASTRO, I., \& UHLIG, S. (2018). Open Connect Everywhere: A Glimpse at the Internet Ecosystem through the Lens of the Netflix CDN. ACM SIGCOMM Computer Communications Review, 48(1), 2834. doi: $10.1145 / 3211852.3211857$

- CAVE, M., \& CROCIONI, P. (2007). Does Europe Need Network Neutrality Rules?. International Journal of Communication, 1, 669-679.

- DONHAM, P. (2010, 15 de agosto). Before Google went evil [web log post]. Recuperado de https://contrarian.ca/2010/08/15/before-google-went-evil/

- FEDERAL COMMUNiCATIONS COMMISSION. (2005, 5 de agosto). New Principles Preserve and Promote the Open and Interconnected Nature of Public Internet. Recuperado de https://bit.ly/2UQpMex 
- FEDERAL COMMUNICATIONS COMMISSION. (2010, 23 de febrero). Report and Order: In the Matter of Preserving the Open Internet, Broadband Industry Practices (GN Docket 09-191, WC Docket 07-52). Recuperado de https://bit. ly/2VBj1ud

- FEDERAL COMMUNICATIONS COMMISSION.(2015, 13 de abril). Protecting and Promoting the Open Internet (GN Docket No. 14-28, FCC 15-24). Recuperado de https://bit.ly/2P6xKLu

- FEDERAL TRADE COMMISSION. (2013, 3 de enero). Google Agrees to Change Its Business Practices to Resolve FTC Competition Concerns In the Markets for Devices Like Smart Phones, Games and Tablets, and in Online Search. Recuperado de http://cort.as/-Gjdi

- FERNÁNDEZ, P. E. (2014). Neutralidad de la red: tensiones para pensar la regulación de Internet. Questión, 1(42), 69-84.

- FONTANALS, G. (2017, abril). La neutralidad de la red y el zero-rating. Revista Fibra. Tecnologías de la comunicación, (14), 1-5. Recuperado de http://papel. revistafibra.info/la-neutralidad-la-red-zero-rating/

- GARCÍA, G. I., \& AGUILAR SÁNCHEZ, C. (2008). Psicoanálisis y política: la teoría de la ideología de Slavoj Žižek. International Journal of Žižek Studies, 2(3), 1-17.

- GENNA, I. (2014, 9 de abril). Expert: The case for search neutrality. EurActiv Special report: Consumer Choice on the Internet. Recuperado de https://bit. ly/2W8RBw2

- GEORGIEVA, I. (2014). Search neutrality as a regulation principle for Internet search engines. A multidisciplinary approach. Master in Intellectual Property Rights, Faculty of Law, Ku Leuven University.

- GLOBAL NET NEUTRALITY COALITION. (s/f). Net neutrality. Recuperado el 21 de abril de 2018 en https://www.thisisnetneutrality.org/

- GOOGLE Inc. (2010, 26 de abril). Reply Comments of Google Inc. before the FCC In the Matter of Preserving the Open Internet Broadband Industry Practices (GN Docket No. 09-191 and WC Docket No. 07-52). 
- GRIMmelmanN, J. (2013). What to Do About Google. Communications of the ACM, 56(9), 28-30. doi: 10.1145/2500129

- HAHN, R., \& WALLSTEN, S. (2006). The economics of net neutrality. Economists' Voice, 3(6), 1-7. doi: 10.2202/1553-3832.1194

- HASTINGS, R. (2014, 20 de marzo). Internet Tolls And The Case For Strong Net Neutrality. Netflix Media Center. Consultado el 16 de abril de 2019 en https://bit. ly/2VCVzzS

- InTERnet ASSOCIATION. (2017). Principles To Preserve \& Protect An Open Internet. Recuperado de https://bit.ly/2UaZxLv

- INTERVOZES \& DERECHOS DIGITALES. (2017). Neutralidad de red en América Latina: reglamentación, aplicación de la ley y perspectivas. Los casos de Chile, Colombia, Brasil y México. Recuperado de https://bit.ly/2D6nApm

- KRÄMER, J., WIEWIORRA, L., \& WEINHARDT, C. (2013). Net neutrality: A progress report. Telecommunications Policy, 37(9), 794-813. doi:10.1016/j. telpol.2012.08.005

- LAMBERT, P. (2010, 24 de febrero). Vodafone and Telefonica overplay their hand with Google. Telecoms.Com. Recuperado de https://bit.ly/2WctKM4

- LOPES MATSUSHITA, T. (2014). El derecho, la sociedad de la información y el principio de la neutralidad de red: consideraciones sobre el mercado y el acceso a la información. Revista de Derecho, Comunicaciones y Nuevas Tecnologías, (11), 1-19. doi:10.15425/redecom.11.2014.02

- MANNE, G., \& WRIGHT, J. (2011). If Search Neutrality is the Answer, What's the Question? (Paper No. 2011-14). Recuperado de https://papers.ssrn.com/sol3/ papers.cfm?abstract_id $=1807951$

- MCCHESNEY, R. W. (2013). Digital disconnect. How capitalism is turning the internet against democracy. New York: New Press. 
- MEDINA, E. (2017, 22 de diciembre). La Neutralidad de Red: nuestra visión [Web log post]. Recuperado de https://bit.ly/2Z2nsQU

- MISENER, P. (2011). Amazon.com: Todos pueden ganar, sin necesidad de hacer concesiones con la neutralidad de red. En J. Pérez Martínez. (Ed.), Neutralidad de red: aportaciones al debate (pp. 137-140). Madrid: Fundación Telefónica - Ariel.

- NEWMAN, R. (2016). The Debate Nobody Knows: Network Neutrality's Neoliberal Roots and a Conundrum for Media Reform. International Journal of Communication, 10, 5969-5988.

- ODLYZKO, A. (2009). Network Neutrality, Search Neutrality, and the Neverending Conflict between Efficiency and Fairness in Markets. Review of Network Economics, 8(1), 40-61. doi:10.2202/1446-9022.1169

- PEPPER, R., \& MAZ, A. (2011). Cisco: Gestionar las redes para un Internet abierto en un mundo de exabytes. En J. Pérez Martínez. (Ed.), Neutralidad de red: aportaciones al debate (pp. 149-152). Madrid: Fundación Telefónica - Ariel.

- SCHNEIBEL, A. G., \& FARIVAR, C. (2010, 4 de julio). Deutsche Telekom moves against Apple, Google and net neutrality. Deutsche Welle. Recuperado de https:// bit.ly/2ZYT4Y9

- SCHTEINGART, D., \& COATZ, D. (2015). ¿Qué modelo de desarrollo para la Argentina?. Boletín informativo TECHINT, 349(2.2), 1. doi:10.13140/ RG.2.1.2258.8642

- WU, T. (2003). Network neutrality, broadband discrimination. Journal of Telecommunications and High Technology Law, 2, 141-179. doi: http://dx.doi. org/10.2139/ssrn.388863

- ZELNICK, B., \& ZELNICK, E. (2013). The illusion of net neutrality: political alarmism, regulatory creep, and the real threat to Internet freedom. Stanford: Hoover Institution Press.

- ZIZEK, S. (2003). El sublime objeto de la ideología. Buenos Aires: Siglo XXI. 


\section{Biografías}

\section{Mariano Zukerfeld}

Universidad de Buenos Aires, Argentina

marianozukerfeld@e-tcs.org

Investigador del CONICET y del equipo e-TCS del Centro CTS (Universidad Maimónides). Doctor en Ciencias Sociales (FLACSO Argentina); Magíster en Ciencia Política y Sociología (FLACSO Argentina), Licenciado en Sociología (UBA). Profesor de la Maestría en Propiedad Intelectual de FLACSO Argentina, de la Maestría en Ciencia Tecnología y Sociedad de la UNQ y de la Carrera de Sociología y del Doctorado de la Facultad de Ciencias Sociales de la UBA. Su último libro es Knowledge in the Age of digital capitalism (2017, University of Westminster Press). De manera general, sus líneas de investigación se vinculan con la relación entre el conocimiento y el capitalismo, con énfasis en la mediación que establece la propiedad intelectual. En particular, indaga en los procesos productivos de software, y el análisis socioeconómico de las tecnologías digitales e internet.

\section{Bernadette Califano}

Universidad de Buenos Aires, Argentina bernacali@gmail.com

Investigadora del Consejo Nacional de Investigaciones Científicas y Técnicas (CONICET) de la Argentina, del equipo e-TCS del Centro CTS de la Universidad Maimónides y del Programa Industrias Culturales y Espacio Público de la Universidad Nacional de Quilmes. Doctora de la Universidad de Buenos Aires en Ciencias Sociales y Licenciada en Ciencias de la Comunicación por la misma casa de estudios. Ha sido becaria doctoral y posdoctoral del CONICET, y becaria Fulbright en la Universidad de California San Diego, Estados Unidos. Profesora de la Facultad de Ciencias Sociales de la UBA, donde dicta asignaturas grado y posgrado sobre políticas de comunicación y tecnologías de la información. Sus líneas de investigación giran en torno de las políticas públicas de comunicación, la convergencia tecnológica, la economía y la regulación de los medios. 


\section{Anexo}

\section{Tabla II}

Velocidades de descarga en internet (Mbps) por banda ancha fija según países (abril 2018)

\begin{tabular}{|c|c|c|c|c|c|}
\hline País & \begin{tabular}{|c} 
Descarga \\
(Mbps)
\end{tabular} & País & $\begin{array}{c}\text { Descarga } \\
\text { (Mbps) }\end{array}$ & País & $\begin{array}{c}\text { Descarga } \\
\text { (Mbps) }\end{array}$ \\
\hline Singapore & 174.94 & Italy & 38.93 & Indonesia & 15.31 \\
\hline Iceland & 162.43 & Ukraine & 36.76 & Kenya & 14.31 \\
\hline Hong Kong (SAR) & 141.05 & Uruguay & 35.55 & $\begin{array}{c}\text { Dominican } \\
\text { Republic }\end{array}$ & 13.97 \\
\hline South Korea & 110.1 & Austria & 34.09 & Albania & 13.93 \\
\hline Romania & 107.01 & Puerto Rico & 33.59 & Morocco & 13.47 \\
\hline Hungary & 94.72 & Qatar & 33.23 & Mauritius & 13.32 \\
\hline Sweden & 93.69 & Panama & 31.88 & Cambodia & 13.23 \\
\hline Switzerland & 93.07 & Belarus & 31.41 & Belize & 12.90 \\
\hline United States & 91.46 & $\begin{array}{c}\text { United Arab } \\
\text { Emirates }\end{array}$ & 30.79 & Côte d'Ivoire & 12.80 \\
\hline Macau (SAR) & 90.29 & Serbia & 30.23 & Azerbaijan & 12.57 \\
\hline Netherlands & 83.24 & Australia & 29.5 & Colombia & 12.55 \\
\hline Norway & 82.22 & Kazakhstan & 27.69 & Guyana & 12.11 \\
\hline Denmark & 81.84 & Malaysia & 26.9 & Angola & 12.01 \\
\hline Canada & 80.08 & Montenegro & 26.58 & Ecuador & 11.88 \\
\hline Japan & 76.31 & Guam & 26.37 & Laos & 11.71 \\
\hline China & 75.43 & Croatia & 25.51 & Maldives & 11.59 \\
\hline Spain & 75.2 & Vietnam & 24.8 & Tanzania & 11.3 \\
\hline New Zealand & 74.22 & Ghana & 23.26 & Iran & 11.06 \\
\hline France & 73.99 & Macedonia & 23.17 & Namibia & 10.05 \\
\hline Jersey & 73.40 & Saudi Arabia & 22.86 & Zimbabwe & 9.72 \\
\hline Lithuania & 70.61 & $\begin{array}{c}\text { Bosnia and } \\
\text { Herzegovina }\end{array}$ & 22.5 & Ethiopia & 9.57 \\
\hline Luxembourg & 64.99 & Mexico & 22.23 & Nigeria & 9.54 \\
\hline Reunion & 64.81 & India & 22.21 & Costa Rica & 9.35 \\
\hline Portugal & 62.54 & Mongolia & 21.87 & Senegal & 9.08 \\
\hline Belgium & 60.61 & Brazil & 21.83 & Syria & 8.61 \\
\hline Germany & 55.7 & Jordan & 21.82 & Iraq & 8.54 \\
\hline Taiwan & 54.06 & Peru & 20.68 & El Salvador & 8.43 \\
\hline
\end{tabular}




\begin{tabular}{|c|c|c|c|c|c|}
\hline Latvia & 52.9 & Curaçao & 20.6 & Myanmar & 8.35 \\
\hline Israel & 52.54 & The Bahamas & 20.58 & French & 8.25 \\
\hline United Kingdom & 51.06 & Cyprus & 20.22 & Tunisia & 7.96 \\
\hline Barbados & 50.61 & Sri Lanka & 20.21 & Pakistan & 7.54 \\
\hline Malta & 49.44 & Jamaica & 19.96 & Guatemala & 7.35 \\
\hline Poland & 45.74 & Armenia & 19.05 & Honduras & 7.32 \\
\hline Slovakia & 45.67 & Bahrain & 18.79 & Paraguay & 7.24 \\
\hline Ireland & 45.08 & Georgia & 18.41 & Libya & 7.1 \\
\hline Finland & 44.85 & Kuwait & 18.34 & Nicaragua & 6.85 \\
\hline Thailand & 44.8 & Argentina & 18.08 & Uzbekistan & 6.78 \\
\hline Bulgaria & 44.55 & Philippines & 17.62 & Bolivia & 6.69 \\
\hline Chile & 43.65 & Greece & 17.53 & Suriname & 6.49 \\
\hline Slovenia & 43.25 & Turkey & 17.41 & Egypt & 6.13 \\
\hline Moldova & 42.8 & Kyrgyzstan & 17.36 & Afghanistan & 6.02 \\
\hline Estonia & 41.82 & Bangladesh & 17.25 & Lebanon & 5.48 \\
\hline Czech Republic & 40.4 & Oman & 17.18 & Algeria & 3.84 \\
\hline Russia & 40.72 & Nepal & 16.12 & Venezuela & 3.73 \\
\hline $\begin{array}{c}\text { Trinidad and } \\
\text { Tobago }\end{array}$ & 40.24 & Fiji & 15.74 & Global & 45.07 \\
\hline
\end{tabular}

Fuente: elaboración propia, con datos de Speedtest.net 
Tabla III

Velocidades de carga y descarga de datos a internet (Mbps) por banda ancha fija según continente (abril 2018)

\begin{tabular}{|c|c|c|c|c|}
\hline Continente & $\begin{array}{c}\text { Carga } \\
\text { (Mbps) }\end{array}$ & $\begin{array}{c}\text { Descarga } \\
\text { (Mbps) }\end{array}$ & $\begin{array}{c}\text { Carga - } \\
\text { Descarga } \\
\text { (Mbps) }\end{array}$ & $\begin{array}{c}\text { Carga - } \\
\text { Descarga (\%) }\end{array}$ \\
\hline Europa & 31.26 & 54.74 & -23.49 & -42.90 \\
\hline $\begin{array}{c}\text { América del } \\
\text { Norte }\end{array}$ & 29.78 & 85.77 & -55.99 & -65.28 \\
\hline Asia & 24.38 & 31.63 & -7.26 & -22.94 \\
\hline Oceanía & 23.13 & 39.82 & -16.69 & -41.92 \\
\hline $\begin{array}{c}\text { Promedio } \\
\text { mundial }\end{array}$ & 21.93 & 45.07 & -23.14 & -51.34 \\
\hline África & 9.80 & 14.48 & -4.67 & -32.29 \\
\hline $\begin{array}{c}\text { América Latina y } \\
\text { el Caribe }\end{array}$ & 7.25 & 19.03 & -11.78 & -61.89 \\
\hline
\end{tabular}

Fuente: elaboración propia, con datos de Speedtest.net ${ }^{17}$

17. Cabe aclarar que esta división por continentes supone el promedio de velocidad de carga y descarga de una cantidad de países muy dispar: Europa incluye datos de 41 países, América del Norte incluye solo a dos (Estados Unidos y Canadá), Asia comprende datos de 92 países, Oceanía de 3, África de 17, mientras que América Latina y el Caribe abarca datos de 28 naciones. 
Tabla IV

Velocidades de carga y descarga de datos a internet por banda ancha fija en países de América Latina y el Caribe (abril 2018)

\begin{tabular}{|c|c|c|c|c|}
\hline País & $\begin{array}{l}\text { Carga } \\
\text { (Mbps) }\end{array}$ & $\begin{array}{l}\text { Descarga } \\
\text { (Mbps) }\end{array}$ & $\begin{array}{c}\text { Carga- } \\
\text { Descarga } \\
\text { (Mbps) }\end{array}$ & $\begin{array}{c}\text { Carga- Descarga } \\
(\%)\end{array}$ \\
\hline Barbados & 29.94 & 50.61 & -20.67 & -40.84 \\
\hline $\begin{array}{c}\text { Trinidad and } \\
\text { Tobago } \\
\end{array}$ & 18.02 & 40.24 & -22.22 & -55.22 \\
\hline Jamaica & 10.53 & 19.96 & -9.43 & -47.24 \\
\hline Belize & 10.43 & 12.90 & -2.47 & -19.15 \\
\hline Brazil & 9.22 & 21.83 & -12.61 & -57.76 \\
\hline Uruguay & 9.14 & 35.55 & -26.41 & -74.29 \\
\hline The Bahamas & 9.01 & 20.58 & -11.57 & -56.22 \\
\hline Chile & 8.80 & 43.65 & -34.85 & -79.84 \\
\hline Ecuador & 8.77 & 11.88 & -3.11 & -26.18 \\
\hline Mexico & 8.23 & 22.23 & -14.00 & -62.98 \\
\hline Puerto Rico & 7.75 & 33.59 & -25.84 & -76.93 \\
\hline Guam & 7.71 & 26.37 & -18.66 & -70.76 \\
\hline Curaçao & 6.98 & 20.60 & -13.62 & -66.12 \\
\hline Panama & 6.71 & 31.88 & -25.17 & -78.95 \\
\hline Colombia & 5.60 & 12.55 & -6.95 & -55.38 \\
\hline Guyana & 5.32 & 12.11 & -6.79 & -56.07 \\
\hline Peru & 4.45 & 20.68 & -16.23 & -78.48 \\
\hline Honduras & 4.34 & 7.32 & -2.98 & -40.71 \\
\hline Paraguay & 3.92 & 7.24 & -3.32 & -45.86 \\
\hline Argentina & 3.84 & 18.08 & -14.24 & -78.76 \\
\hline Dom. Republic & 3.68 & 13.97 & -10.29 & -73.66 \\
\hline Nicaragua & 3.64 & 6.85 & -3.21 & -46.86 \\
\hline Costa Rica & 3.60 & 9.35 & -5.75 & -61.50 \\
\hline El Salvador & 3.29 & 8.43 & -5.14 & -60.97 \\
\hline Guatemala & 3.15 & 7.35 & -4.20 & -57.14 \\
\hline Bolivia & 2.92 & 6.69 & -3.77 & -56.35 \\
\hline Suriname & 2.67 & 6.49 & -3.82 & -58.86 \\
\hline Venezuela & 1.33 & 3.73 & -2.40 & -64.34 \\
\hline
\end{tabular}

Fuente: elaboración propia, con datos de Speedtest.net 


\section{Tabla V}

Velocidades de carga y descarga de datos a internet en países con velocidad de carga superior a la de descarga (abril 2018)

\begin{tabular}{|c|c|c|c|c|}
\hline País & $\begin{array}{c}\text { Carga } \\
\text { (Mbps) }\end{array}$ & $\begin{array}{c}\text { Descarga } \\
\text { (Mbps) }\end{array}$ & $\begin{array}{c}\text { Carga- } \\
\text { Descarga } \\
\text { (Mbps) }\end{array}$ & $\begin{array}{c}\text { Carga- Descarga } \\
\text { (Mbps }\end{array}$ \\
\hline Japón & 89.21 & 76.31 & 12.90 & 16.90 \\
\hline Singapur & 185.45 & 174.94 & 10.51 & 6.01 \\
\hline Islandia & 170.65 & 162.43 & 8.22 & 5.06 \\
\hline Etiopia & 14.26 & 9.57 & 4.69 & 49.01 \\
\hline Cambodia & 15.29 & 13.23 & 2.06 & 15.57 \\
\hline Iraq & 9.74 & 8.54 & 1.20 & 14.05 \\
\hline Bangladesh & 18.08 & 17.25 & 0.83 & 4.81 \\
\hline Fiji & 16.45 & 15.74 & 0.71 & 4.51 \\
\hline Afganistán & 6.45 & 6.02 & 0.43 & 7.14 \\
\hline Mongolia & 22.22 & 21.87 & 0.35 & 1.60 \\
\hline Kyrgyzstan & 17.66 & 17.36 & 0.30 & 1.73 \\
\hline Ucrania & 36.86 & 36.76 & 0.10 & 0.27 \\
\hline Georgia & 18.50 & 18.41 & 0.09 & 0.49 \\
\hline
\end{tabular}

Fuente: elaboración propia, con datos de Speedtest.net 
Tabla VI

Tres posicionamientos respecto de la neutralidad de la red

\begin{tabular}{|c|c|c|}
\hline Posicionamiento & Actores políticos & $\begin{array}{l}\text { Lineamientos de } \\
\text { política pública }\end{array}$ \\
\hline $\begin{array}{c}\text { En contra de la } \\
\text { neutralidad de la red }\end{array}$ & $\begin{array}{l}\text { Grandes empresas de } \\
\text { infraestructura }\end{array}$ & $\begin{array}{c}\text { Introducir discriminacio- } \\
\text { nes de precios, rechazar } \\
\text { regulaciones }\end{array}$ \\
\hline $\begin{array}{c}\text { A favor de la neutralidad } \\
\text { de la red }\end{array}$ & $\begin{array}{c}\text { Grandes firmas de } \\
\text { software y contenidos }\end{array}$ & $\begin{array}{l}\text { Rechazar discriminacio- } \\
\text { nes de precios pero evadir } \\
\text { otras regulaciones que } \\
\text { nivelarían el terreno }\end{array}$ \\
\hline $\begin{array}{l}\text { Neutralidad desarrollista: } \\
\text { tercera posición }\end{array}$ & $\begin{array}{l}\text { Actores sin fines de lucro, } \\
\text { pymes y usuarios de } \\
\text { internet, especialmente } \\
\text { aquellos de países } \\
\text { periféricos }\end{array}$ & $\begin{array}{c}\text { Garantizar efectivamente } \\
\text { acceso igualitario para } \\
\text { todos los usuarios de } \\
\text { internet } \\
\text { Intervención estatal: } \\
\text { nivelar el terreno a través } \\
\text { de políticas que tengan en } \\
\text { cuenta, por ejemplo, los } 5 \\
\text { temas aquí desarrollados }\end{array}$ \\
\hline
\end{tabular}

Fuente: elaboración propia 